\title{
An Optimal Framework for WDM Systems Using Analytical Characterization of Refractive Index-Related Nonlinear Impairments
}

\author{
Muhammad Irfan 1,*(D), Farman Ali ${ }^{2, *(\mathbb{D})}$, Fazal Muhammad ${ }^{3, *(\mathbb{D}}$, Abdullah S. Alwadie 1(D), Adam Glowacz ${ }^{4, *(\mathbb{D})}$ \\ Iwona Goldasz ${ }^{5}$, Ryszard Mielnik ${ }^{6}$, Fahad Salem Alkahtani ${ }^{1}$ and Hidayatullah Khan ${ }^{7}$
}

\section{check for}

updates

Citation: Irfan, M.; Ali, F.; Muhammad, F.; Alwadie, A.S.; Glowacz, A.; Goldasz, I.; Mielnik, R.; Alkahtani, F.S.; Khan, H. An Optimal Framework for WDM Systems using Analytical Characterization of Refractive Index Related Nonlinear Impairments. Electronics 2021, 10, 221 https://doi.org/10.3390/electronics 10030221

Received: 15 November 2020 Accepted: 21 December 2020 Published: 20 January 2021

Publisher's Note: MDPI stays neutral with regard to jurisdictional clai$\mathrm{ms}$ in published maps and institutional affiliations.

Copyright: (C) 2021 by the authors. Licensee MDPI, Basel, Switzerland. This article is an open access article distributed under the terms and conditions of the Creative Commons Attribution (CC BY) license (https:// creativecommons.org/licenses/by/ $4.0 /)$.
1 Electrical Engineering Department, College of Engineering, Najran University Saudia Arabia, Najran 61441, Saudia Arabia; asalwadie@nu.edu.sa (A.S.A.); fsalkahtani@nu.edu.sa (F.S.A.)

2 Department of Electrical Engineering, Qurtuba University of Science and IT, D. I. Khan 29050, Pakistan

3 Department of Electrical Engineering, University of Engineering Technology, Mardan 23200, Pakistan

4 Department of Automatic Control and Robotics, Faculty of Electrical Engineering, Automatics, Computer Science and Biomedical Engineering, AGH University of Science and Technology, al. A. Mickiewicza 30, 30-059 Kraków, Poland

5 Faculty of Environmental and Power Engineering, Cracow University of Technology, Warszawska 24 Str., 31-155 Cracow, Poland; iwona.goldasz@pk.edu.pl

6 Faculty of Electrical and Computer Engineering, Cracow University of Technology, Warszawska 24 Str., 31-155 Cracow, Poland; ryszard.mielnik@pk.edu.pl

7 Department of Electrical Engineering, CUI, Wah Campus Islamabad, Islamabad 44000, Pakistan; hidayat.msk@gmail.com

* Correspondence: miditta@nu.edu.sa (M.I.); drfarmanali.optics@qurtuba.edu.pk (F.A.); fazal.muhammad@uetmardan.edu.pk (F.M.); adglow@agh.edu.pl (A.G.)

\begin{abstract}
Nonlinear effects in the optical transmission systems (OTSs) are considered as the major performance limiting factor to provide high transmission rates over ultra-long distances. As the demands for system capacity, transmission range and the number of users is increasing exponentially with the development of mobile broadband, new challenges are being faced by the backbone optical networks. Mainly, the refractive index related non-linearities (RIrNLs) need to be characterized to design an optimal OTS for error-free transmission with provision of wavelength division multiplexing (WDM) to support for multiple channels. This paper provides an estimation technique of RIrNLs for long-haul transmission and their treatment for different channel spacing and the number of channels in a WDM system operating frequency domain multiple in multiple out (FD-MIMO) equalizer based digital signal processing (DSP) receiver and microstrip Chebyshev low pass filter. The main focus of this work is to utilize the existing structure of OTS for RIrNLs treatment with a low cost solution. Thus, by varying the parameters of the third order dispersion parameters, group velocity dispersion parameters, phase modulation dispersion and nonlinear refractive index, the impact of RIrNLs is investigated in detail to enhance the transmission range and capacity of the current OTS. The proposed system is analyzed in terms of range of input power, fiber length and received power for OTS figure of merits including bit error rate (BER) and optical signal-to-noise ratio (OSNR). Using duo-binary modulation, the BER achieved in this work is $<10^{-5}$ till $500 \mathrm{~km}$ range, for maximum number of 32 channels, with $100 \mathrm{Gbps}$ aggregate data rate, which shows the feasibility and effectiveness of our proposed model.
\end{abstract}

Keywords: duo-binary modulation; refractive index related non-linearities; frequency domainmultiple in multiple out (FD-MIMO) equalizer-based DSP; microstrip Chebyshev low pass filter; channel spacing 


\section{Introduction}

Nonlinear effects in long haul optical transmission systems (OTSs) are the major limitations on the high capacity, long haul and ultra-reliable transmission [1]. The performance of a simple OTS is severly affected by the non-linear effects, which are developed when high capacity signals are communicated over extensive distances [2,3]. As a result, the performance is degraded, which leads to errors and data integrity issues. These nonlinear effects can be categorized into two types. The first is refractive index-related nonlinearities (RIrNLs), such as cross phase modulation (XPM) [4], self phase modulation (SPM) [5], and four wave mixing (FWM) [6]. The second type of non-linearities are scattering-related, which includes stimulated Raman scattering (SRS) [7] and stimulated berillouin scattering (SBS) [8]. The refractive index-related nonlinearities (RIrNLs) generate unwanted frequencies inside the optical medium, due to mixing of transmitted optical signals with each other or with the carrier wavelength during their propagation. Instead of using multiple fibers [9], the transmission of multiple data channels [10] is always desirable but the extra signals inside the single mode fiber (SMF) cause power penalty problems in long-haul cases because of the erbium doped fiber amplifier (EDFA) saturation in each transmission span and channel filtering at the receiver in a wavelength division multiplexing (WDM) system.

\subsection{Related Work}

The main features of OTSs are high capacity and long-haul transmission due to huge amount of available bandwidth and extremely low propagation loss. To ensure transmission integrity, a large volume of literature exists on treatment of channel effects. Some advanced contributions and their limitations are presented as follow. Digital signal processing techniques for compensating inter-and intra-channel fiber non-linearities have been investigated in [11], and input parameters such as launch power and fiber length are used to carry out simulation work. Self phase modulation (SPM) non-linear issue is investigated in [12] where conversion between different formats, such as 8-quadrature amplitude modulation (8-QAM) to quadrature phase shift keying (QPSK) and on off keying (OOK), is performed through optical methods to adapt to the channel conditions. Different values of optical signal-to-noise ratio (OSNR), optical power, and chromatic dispersion have been used to conduct analysis on the performance of the proposed system, however, the format conversion to a lower order results in decreased data rates. In [13], the authors discuss intermodel non-linear issues in few-mode fiber using 16-QAM up to $36 \mathrm{~km}$ transmission and report mitigation of the XPM non-linear effect. High cost components are used in [14], such as indium phosphate and silicon photonics to achieve high bandwidth in the presence of fiber non-linearities. The voltra-assisted method is explained in [15], in which the authors overcome the limitations of optical phase conjugation (OPC) technique. In [16], the authors mitigate nonlinearity for 92-channel 22.5 buad rate, using 16-QAM modulation format. Similarly, SPM and XPM nonlinear parameters are discussed in [17], implementing 16-QAM and 64-QAM super channels and advanced equalization procedures. In [18], linearly swept optical source methodology is developed for mitigating high order FWM effects. The impact of nonlinearity inside optical fiber is investigated by spectral efficient frequency division multiplexing (SEFDM) technique and the work is compared with orthogonal-FDM (O-FDM). In [19], nonlinear mitigation technique is reported for multi subcarrier PM-16QAM using symbol rate optimization in 31 channel WDM system. In [20], the authors have explored nonlinear compensation mechanism with the help of multi-channel digital back propagation in Nyquist spaced optical communication system. However, the effect of RIrNLs still needs to be investigated, in terms of analytical characterization, to understand their effect to achieve an optimum mitigation solution, which is the main focus of our paper. Moreover, modern systems require multiplexing of several channels for which different parameters for a WDM based system need to be analyzed in this context. Therefore, we present a detailed analysis of RIrNLs and their treatment by varying input power, fiber length, channel spacing, and the number of channels. We present a WDM system with a $100 \mathrm{~Gb} / \mathrm{s}$ aggregate data rate, which can support transmission distances up to $500 \mathrm{~km}$, 
with 32 number of channels. The performance analysis has been conducted for a range of non-linear distortions. To the best of our knowledge, such performance has only been possible with the mathematically proposed mitigation technique for second and third order non-linearities.

\subsection{Major Contributions}

The major contributions of this paper are listed below.

1. Transmission distances of up to $500 \mathrm{~km}$ have been used in this work and the corresponding nonlinear effects have been investigated.

2. The proposed system is analyzed through an analytical and simulation model. The characterization is presented through a mathematical model and an optimal design is proposed using frequency domain-multiple in multiple out (FD-MIMO) equalizer and microstrip chedyshev low pass filter.

3. The parameters used for simulation analysis include channel spacing, nonlinear refractive index, fiber length, input power, and nonlinear dispersion, to ensure a comprehensive analysis.

4. The performance of the proposed model is quantified in terms of BER and OSNR, where these two parameters are achieved below $10^{-9}$ and above 25 , respectively.

5. FWM, XPM and SPM are reduced successfully till $500 \mathrm{~km}$ covered distance for channel spacing as low as $25 \mathrm{GHz}$ and number of channels as much as 32 .

The OptiSystem software package, used in this work for simulation investigations, provides a library of multiple optical components that enables users to plan, test, and simulate different types of OTSs. The modeling of end-to-end downlink transmission system in this paper has been performed by the characterization of each component, careful investigation of interdependencies among different components, modeling of noise for active components in the system and other considerations to realize a practical OTN. The varying refractive index response and intrinsic group velocity delay for SMF, and DSP block are modeled in MATLAB and integrated with the optisystem.

The remainder of this work is arranged as follows. Section 2 presents the proposed system model layout, followed by the description of analytical model in Section 3. Section 4 summarizes results and discussion of the presented system. Finally, the conclusion is presented in Section 5.

\section{Proposed Model Layout}

This section clarifies the framework of the proposed system and performance against RIrNLs. The increase in transmission channels gives rise to further distortion effects including channel-to-channel interference or beating noise, saturation of EDFA due to distribution of the overall transmit power to multiple channels, wavelength dependent chromatic dispersion interaction with several wavelengths as compared to the single channel. The WDM system is idealized by using 4 to 32 number of continuous wave (CW) laser sources to investigate the corresponding increase in non-linear effects due to the multiple channel transmission. An advanced duo-binary modulation technique is implemented at the transmitter side, whose function is to adjust the spectral width of the input optical signal, as presented in Figure 1. Each channel is modulated with a $3.125 \mathrm{~Gb} / \mathrm{s}$ data rate signal which provides an aggregate data rate of $100 \mathrm{~Gb} / \mathrm{s}$ for 32 numbers or channels.

Duo-binary modulation is implemented by using an electrical time delay line with an exclusive OR gate, which encloses pseudo-random binary sequence (PRBS) generator and uses two LiNb Mach-Zehnder modulators (MZMs) as a simulation model in Optisystem software. This model implements duo-binary modulator through a duo-binary precoder, an electrical subtractor, $\chi$, and delay, $\tau$, to generate duo-binary signal. After modulation from each channel, the data are multiplexed using dense wavelength division multiplexer (DWDM) which has $-100 \mathrm{~dB}$ threshold noise and $3 \mathrm{~dB}$ dynamic noise. After multiplexing, optical signals are transmitted over SMF. Above $500 \mathrm{~km}$, SMF is used with five spans 
of $100 \mathrm{~km}$ each. To minimize RIrNLs, linear dispersion and attenuation, each span is connected with EDFA and semiconductor optical amplifier (SOA). At the receiver, $R_{x}$, side, the data is de-multiplexed (DeMUX) and optical signals are converted to electrical form using avalanche photo diode (APD) with $1 \mathrm{~A} / \mathrm{W}$ responsivity and $100 \times 10^{-24} \mathrm{~W} / \mathrm{Hz}$ thermal power density. The gain of diode is maximized several times and enables greater sensitivity as compare to conventional photo diode (PD). The signal then proceeds to microstrip Chebyshev low pass filter to minimize the factors of chromatic dispersion (CD), and amplified spontaneous noise (ASE). The filtered signals are then purified from RIrNLs by employing limiting RF amplifier and enhanced DSP system. The proposed DSP receiver includes a FD-MIMO equalizer, synchronizing block to balance the flow of multiple received wavelengths and time domain equalizer (TDE) to support the phase behavior of the received signals. The internal procedure of FDE-MIMO equalizer is presented in Figure 2, employing overlap save technique . Moreover, 3R-regenerator component is installed for re-shaping, re-timing and re-amplification of the received signals, where they are linked to a BER analyzer to investigate the received pulse property. The list of parameters with their values for the simulation are described in Table 1. As compared to [19-21], the authors have investigated the performance of duo-binary modulation for accumulative distortion effects caused due to transmission of large number of WDM channels over long-haul distances, while varying the wavelength dependent chromatic dispersion and refractive index of the optical fiber.

Table 1. Simulation parameters with their values and units.

\begin{tabular}{cc}
\hline Description & Magnitude \\
\hline Reference wavelength & $1550 \mathrm{~nm}$ \\
\hline Attenuation & $0.2 \mathrm{~dB} / \mathrm{km}$ \\
\hline Input power & $-6 \mathrm{dBm}$ to $6 \mathrm{dBm}$ \\
\hline Received power & $-27 \mathrm{dBm}$ to $-16 \mathrm{dBm}$ \\
\hline Channel spacing & $50 \mathrm{GHz}$ to $200 \mathrm{GHz}$ \\
\hline Length of fiber & $500 \mathrm{~km}$ \\
\hline Nonlinear effective area & $80 \mu \mathrm{m}^{2}$ \\
\hline Number of Channel & $4-32$ \\
\hline Nonlinear dispersion & $-3 \mathrm{ps}^{3} / \mathrm{km}$ \\
\hline
\end{tabular}

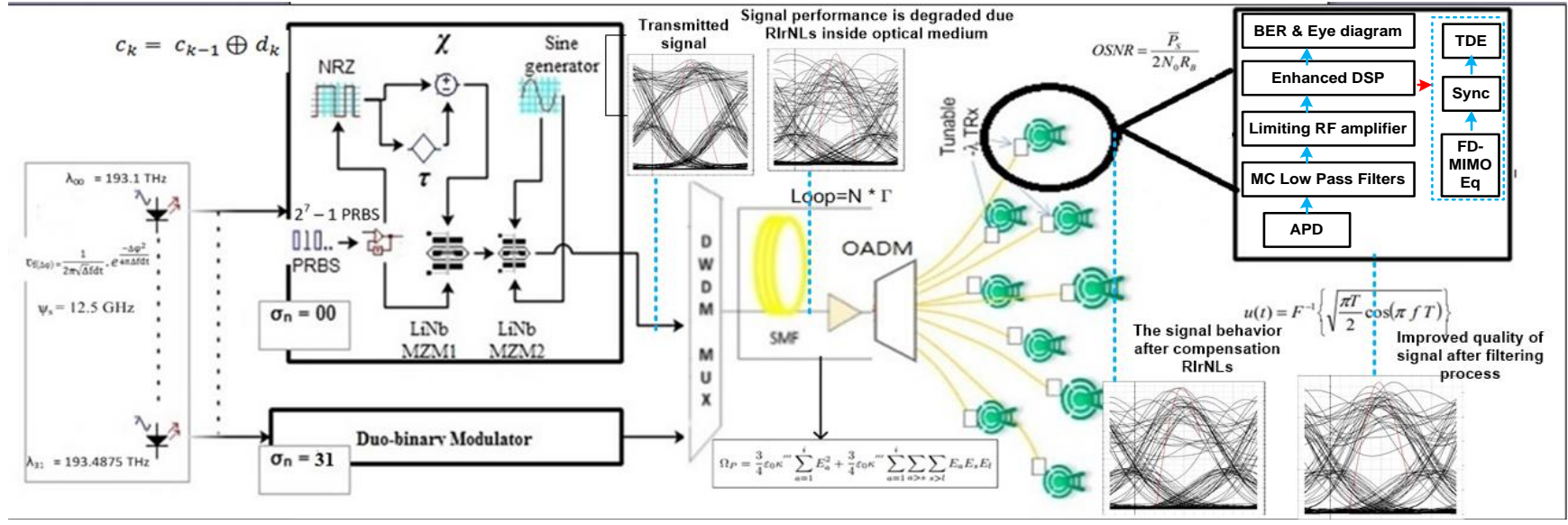

Figure 1. WDM based OTN model for mitigation of RIrNLs. 


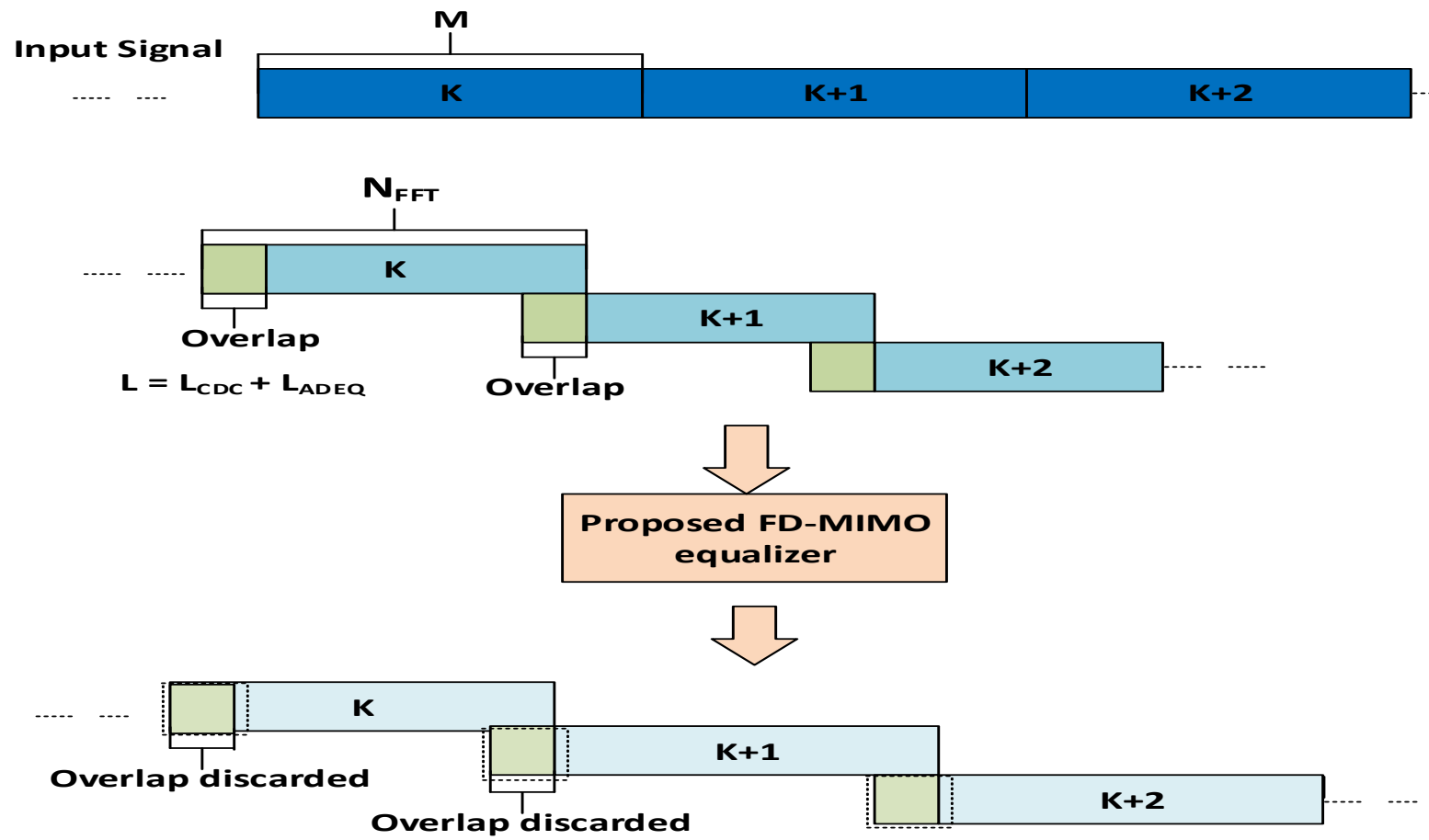

Figure 2. Procedure of frequency domain multiple in multiple out (FD-MIMO) equalizer.

\section{Refractive Index Related Non-Linearities}

The induced non-linearities in optical systems are composed of two types; the first type relates to nonlinearities due to scattering phenomena [19], and the second type of nonlinearities are associated with refractive index of the optical fiber. The second type of nonlinearities are known as RIrNLs. FWM, XPM and SPM nonlinear factors are the main subparts of RIrNLS. A brief explanation of each nonlinear parameter is given below.

Four wave mixing (FWM) is an intermodulation incident that occurs inside an optical fiber [20], whereby interaction among the channel frequencies generate new unwanted frequencies, as shown in Figure 3. It is particularly important for WDM-based OTN, where multiple wavelengths travel through the same fiber. From Figure 3, it is clear that $\vartheta_{f 00}, \vartheta_{f 01}$, and $\vartheta_{f 02}$ are induced unwanted pulses with original $\vartheta_{f i i}, \vartheta_{f i j}$ and $\vartheta_{f k k}$ imparted optical signals, respectively. FWM nonlinearity is generated due to the third order susceptibility [21], when high power is launched to ensure the propagation of high capacity optical signals over long distances. The distortion caused by FWM is worse than the other two subtypes of RIrNLs.

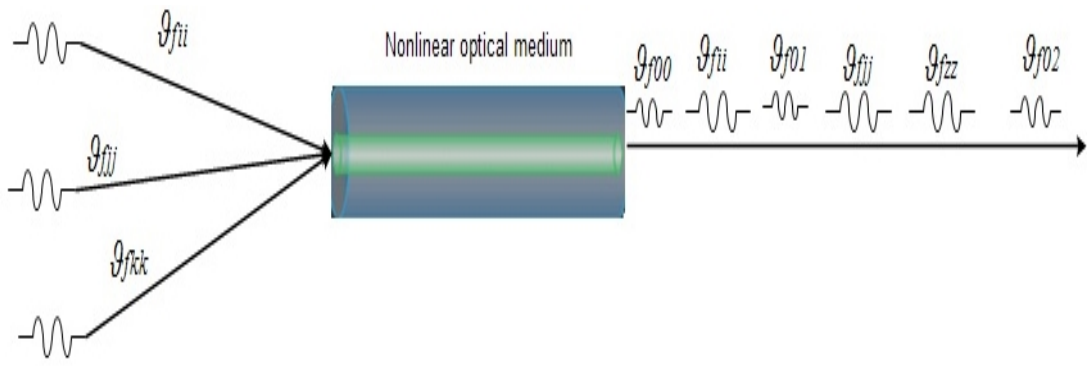

Figure 3. Induction of unwanted frequencies due to four wave mixing (FWM) effect.

Another phenomena which is important for WDM-based systems is the cross phase modulation (XPM) which happens due to variation in refractive index of the optical fiber 
along the transmission length. Phase shift in the optical pulse is induced due to alternating refractive index of the fiber as the signal travels through it. This phenomenon leads to a change in optical signal frequency spectrum as shown in Figure 4. This effect is directly proportional to the number of channels passing inside the SMF [22]. Figure 4 depicts that the output signal is disturbed due to XPM factor. As the number of channels in a WDM framework changes dynamically, an adaptive equalization is required to cancel the XPM influence on optical signals.
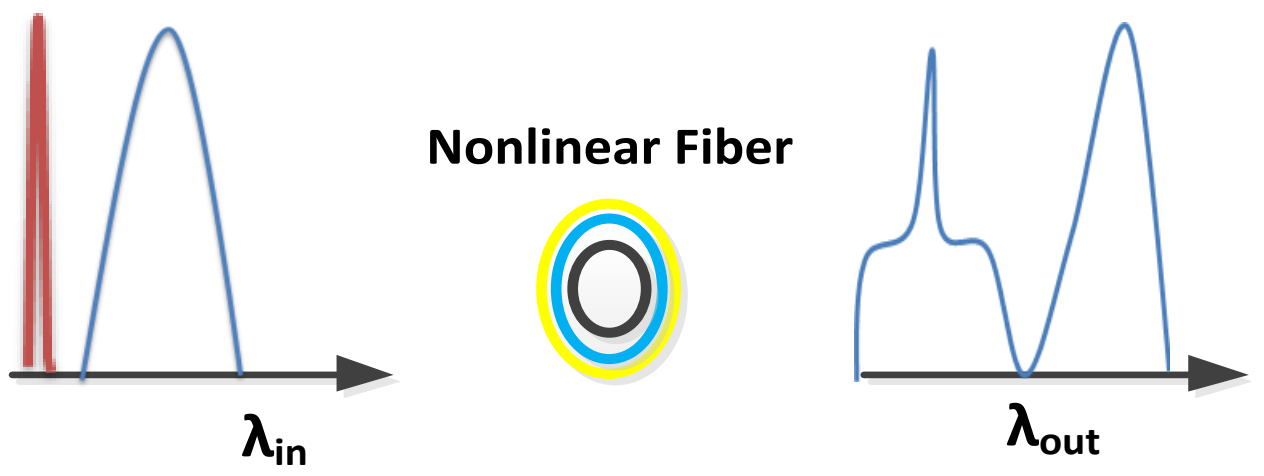

Figure 4. Cross phase modulation (XPM) effects inside nonlinear optical medium.

An associated kind of RIrNLs is the self phase modulation (SPM), caused due to the self modulation of an optical signal in a SMF. It leads to spectral broadening [23] and phase delay [24], as shown in Figure 5. Though it is not directly caused by the WDM transmission, the effect of SPM increases with the launched power levels and the optical pulse spreads and tends to overlap the neighboring optical channels. Consequently, a particular signal of interest is not distinguished by the receiver for WDM channels. Moreover, the penalty in power is also caused on account of SPM.
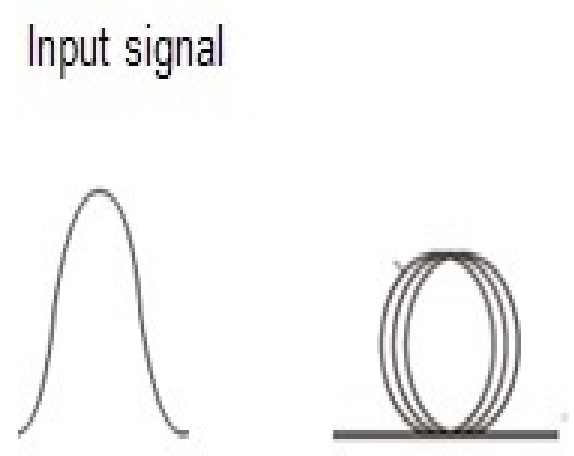

Signal

\section{affected due to}

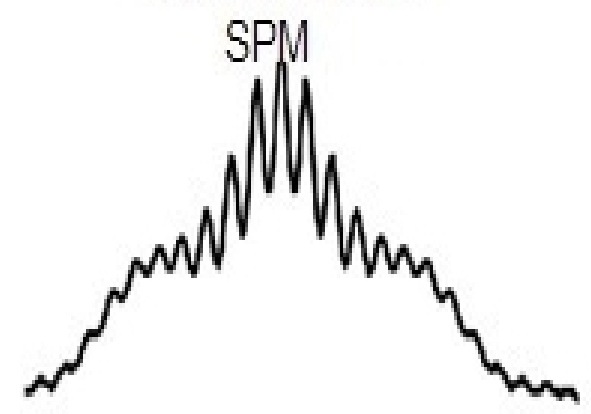

Figure 5. Pulse broadening due to self phase modulation (SPM) non-linearity.

XPM, SPM and FWM are collectively addressed as RIrNs, because these are generated due to the variation in the refractive index. As these effects have strong contribution in degradation of a WDM system, the proposed model aims to minimize them through mathematical modeling and verification through simulations. Features, such as duo-binary modulation, channel spacing, nonlinear effective area and refractive index, and nonlinear dispersion are discussed in this paper to investigate RIrNLs. This section demonstrates the framework of the proposed work, while the next section concentrates on the analytical model and the proposed solution.

\section{Analytical Modeling}

The proposed system aims to maintain system fidelity for long distance transmission, system volume and data rates. This section presents a detailed analytical model for 
analyzing RIrNLs. The parameters which affect the amount of RIrNLs are cross-section area of SMF, amount of light intensity propagating through the fiber, number of channels in the WDM system, and data rate. These factors generate an-harmonic motions of photons inside the SMF [25], where the behavior of induced polarization $\Omega_{P}$ no more remains linear, which is given as

$$
\Omega_{P}=\varepsilon_{0} \kappa^{\prime} E+\varepsilon_{0} \kappa^{\prime \prime} E^{2}+\varepsilon_{0} \kappa^{\prime \prime \prime} E^{3} .
$$

Here, $\varepsilon_{0}$ describes free space permittivity, $E$ means electrical field intensity, and $\kappa^{\prime}$, $\kappa^{\prime \prime}$ and $\kappa^{\prime \prime \prime}$ determine first, second and third order susceptibility, respectively.

The effects of RIrNLs for optical medium are originated from the electric diplole polarization induction, which is mainly due to the $\kappa^{\prime}$, while the $\kappa^{\prime \prime}$ does not contribute for silica based propagation mediums [26] and $\kappa^{\prime \prime \prime}$ produces very low order nonlinear effects. Thus, by neglecting $\kappa^{\prime \prime}$, the induced polarization from (1) becomes

$$
\Omega_{P}=\varepsilon_{0} \kappa^{\prime} E \cos (\Phi t-z x)+\varepsilon_{0} \kappa^{\prime \prime \prime} E^{3} \cos ^{3}(\Phi t-z x) .
$$

In (2), both linear and nonlinear parameters are included.

For the phase wave explored in (2), the intensity $\iota$ of pulse [27] is defined as

$$
\iota=\frac{1}{2} c \varepsilon_{0} n_{0} E_{0}^{2}
$$

Here, $c$ denotes the light velocity, and linear refractive index is represented by $n_{0}$ at low electrical fields [28]. Thus, (2) simplifies to

$$
\Omega_{P}=\varepsilon_{0} \kappa^{\prime} E \cos (\Phi t-z x)+\frac{3}{2} \frac{\kappa^{\prime \prime \prime}}{c \varepsilon_{0} n_{0}} \iota E \cos (\Phi t-z x),
$$

The effective susceptibility, $\kappa_{e}$, of the optical medium is calculated as

$$
\kappa_{e}=\kappa^{\prime}+\frac{3}{2} \frac{\kappa^{\prime \prime \prime}}{c \varepsilon_{0} n_{0}} \iota
$$

and the effective refractive index, $n_{e}$, is measured as

$$
n_{e}=n_{0}+n_{r} \iota
$$

In (6), $n_{r}$ represents nonlinear refractive index, which is given as

$$
n_{r}=\frac{3}{4} \frac{\kappa^{\prime \prime \prime}}{c \varepsilon_{0} n_{0}^{2}}
$$

In addition, RIrNLs enlarge with the increase in length of fiber. As length of fiber gets larger, the interaction of a signal with the fiber and other traveling signals increases, which results in enhancing the effect of RIrNLs [28]. This optical power, in terms of length of fiber, $\Gamma$, and attenuation, $\alpha$, is written as

$$
\rho_{r}=\rho_{i} \exp ^{-\alpha \Gamma}
$$

where $\rho_{i}$ and $\rho_{r}$ determine input and output powers. For length, $\Gamma$, the effective length, $\Gamma_{e}$, of the fiber is given as

$$
\rho_{i} \Gamma_{e}=\int_{\Gamma=0}^{\Gamma=w} \rho_{r}(\Gamma) d \Gamma .
$$

From (8) and (9), $\Gamma_{e}$ is written as

$$
\Gamma_{e}=\frac{1-\exp ^{-\alpha \Gamma}}{\alpha}
$$


The effective length after installing optical amplifiers with $\Gamma$ and amplifier spaced distance $z$ [29], is defined as

$$
\Gamma_{e}=\frac{1-\exp ^{-\alpha \Gamma^{2}}}{\alpha} \frac{\Gamma}{z} .
$$

Cross sectional area, $\xi_{e f f}$, of fiber core has inverse relation to $\iota[30]$ and can be estimated as

$$
\xi_{e f f}=\frac{\int_{m} \int_{b} m d m d b}{\int_{m} \int_{b} m d m d b \iota(m, b)}
$$

where $m$ and $b$ are the polar coordinates. Due to the SPM, different parts of the optical signal undergo different phase shifts, which results in frequency chirping incident. Broadening of spectrum is the initial impact of SPM, which gets larger for high launch power. Hence, based on wavelength, $\lambda, \Gamma$ and $E$, the phase, $\omega,[31]$ is defined as

$$
\omega=\frac{2 \pi}{\lambda} n \Gamma \text {. }
$$

Here, $n$ represents refractive index. In terms of linear and nonlinear phenomena, (13) is written as

$$
\omega=\frac{2 \pi}{\lambda}\left(n_{0}+n_{r} \iota \Gamma_{e}\right) .
$$

The first portion of the above equation is called linear phase $\omega_{0}$, and the second portion is known as nonlinear phase shift $\omega_{r}$. For time dependent phase angle, the modulated optical carrier frequency, $\vartheta_{c}$, based on frequency spectrum, $\vartheta_{0}$, Ref. [32] is given as

$$
\vartheta_{c}=\vartheta_{0}+\frac{d \omega}{d t} .
$$

The SPM is a type of RIrNLs which causes fluctuations in the transmitted signal. In addition, XPM is another major RIrNL which happens when multi optical signals propagate over fiber simultaneously. For this effective refractive index, $n_{e}$ is expressed as

$$
n_{e}=n_{0}+n_{r} \frac{\rho_{i}}{\xi_{e}}
$$

Due to XPM, propagation constant $H$ becomes power dependent, and is defined as

$$
H=H_{0}+H_{r} \rho,
$$

where $H_{0}$ is linear propagation constant and $H_{r}$ is nonlinear propagation constant. Furthermore, the nonlinear phase shift, $\omega_{r}$, is calculated as

$$
\omega_{r}=\int_{0}^{\Gamma}\left(H_{e}-H_{0}\right) d m
$$

When multiple channels are transmitted simultaneously, the nonlinear phase shift in a signal is related with co-channels. Therefore,

$$
\omega_{r}=H_{e} \Gamma_{e}\left(\rho_{v 0}+2 \rho_{v 1}\right),
$$

where $\rho_{v 0}$ and $\rho_{v 1}$ are the powers of two co-channels. This equation demonstrates that XPM degrades the system reliability several folds as compared to SPM.

From (1) and (2), it is described that induced polarization composes of both linear and RIrNLs terms. The FWM nonlinear impact is caused by the third order susceptibility. In this process, photons of transmitted signals annihilate and result in production of new photons at various frequencies. In contrast to SPM and XPM, FWM is independent of bit 
rate and dependent on channel spacing and fiber dispersion. To calculate the effect of FWM, the induced polarization is written as

$$
\begin{aligned}
& \Omega_{P}=\frac{3}{4} \varepsilon_{0} \kappa^{\prime \prime \prime} \sum_{a=1}^{i} E_{a}^{2}+\frac{3}{4} \varepsilon_{0} \mathcal{K}^{\prime \prime \prime} \sum_{a=1}^{i} \sum_{a>s} \sum_{s>l} E_{a} E_{s} E_{l} \\
& \cos \left(2 \vartheta_{i i}+\vartheta_{j j}+\vartheta_{k k}\right) t-\left(H_{i i}+H_{j j}+H_{k k}\right) z \\
& +\cos \left(\vartheta_{i i}+\vartheta_{j j}+\vartheta_{k k}\right) t-\left(H_{i i}+H_{j j}+H_{k k}\right) z \\
& +\cos \left(\vartheta_{i i}-\vartheta_{j j}+\vartheta_{k k}\right) t-\left(H_{i i}-H_{j j}+H_{k k}\right) z \\
& +\cos \left(\vartheta_{i i}-\vartheta_{j j}-\vartheta_{k k}\right) t-\left(H_{i i}-H_{j j}-H_{k k}\right) z .
\end{aligned}
$$

The primary portion in the above equation represents SPM and XPM, while the secondary part denotes FWM. It explains that FWM occurs due to the mixing of different frequencies. As RIrNLs are the main causes of power penalty and system performance deduction, the proposed system mitigates their effects by employing NRZ duo-binary modulation, large effective area and uneven channel spacing. Moreover, RIrNLs can be limited by controlling nonlinear dispersion through a dispersion compensation fiber (DCF). The BER is used for the analysis of RIrNLs and phase noise parameters; this means that if these issues exist, then we do not know how many bits can be transferred including low error size per bit. BER is given as

$$
\mathrm{BER}=\frac{1}{2 \operatorname{erf} \sqrt{\frac{\mathrm{OSNR}}{2}}}
$$

The erf denotes error function the optical signal to noise ratio (OSNR) is described as

$$
O S N R=E_{S N R} \frac{\varphi}{2 B_{r} \cdot m_{\text {sys }}}
$$

where $B_{r}$ defines the reference bandwidth, $E_{S N R}$ is the electrical SNR, $\varphi$ is the total transmission symbol rate measured in Gbps and the last term $m_{s y s}$ describes the system margin.

\section{Results and Discussion}

This section elaborates the simulation results of the proposed system. Optisystem Ver.13 simulation software is used to carry out the simulations, which contains most of the components used for the design of a practical optical communication system. The performance metric of BER, specified by the number of error bits per transmitted ones, is performed at the receiver end in Optisystem by using a clock recovery module, followed by a data recovery component. The Optisystem makes use of the sequence length and makes several iterations to estimate the BER values. The threshold of $10^{-3}$ is used in this work as mentioned by a dotted line in the figures containing results. The BERs of eye diagrams are found to be higher than the considered threshold value before Nonlinearities compensation, but is much lower $\left(\mathrm{BER}<10^{-5}\right)$ with application of the proposed compensation technique. In order to discuss pre-FEC BER, the optical transmission system interfaces on series of packet transport routers support monitoring the condition of an optical network by using pre-FEC BER. In the Optisystem, BER is estimated using a Gaussian algorithm with transmission of a short sequence of bits. After the bit recovery, the BER test set calculates the error bits and use the transmitter channel information to produce the BER. Figure 6 describes the flow chart for connection among analytical and simulation models.

Figure 7 presents the BER performance of the system against the effective non-linear area of the optical fiber. The simulations are conducted for different channel spacings used for the proposed WDM system. The results show that the performance of the system against RIrNLs improves with the increase in area and is optimum at $80 \mu \mathrm{m}^{2}$ effective area. Similarly, Figure 8 presents the OSNR results for different channel spacings, as the effective 
area is varied. The simulation results are plotted for a 32-channel WDM system, with an aggregate data rate of 100 Gbps.

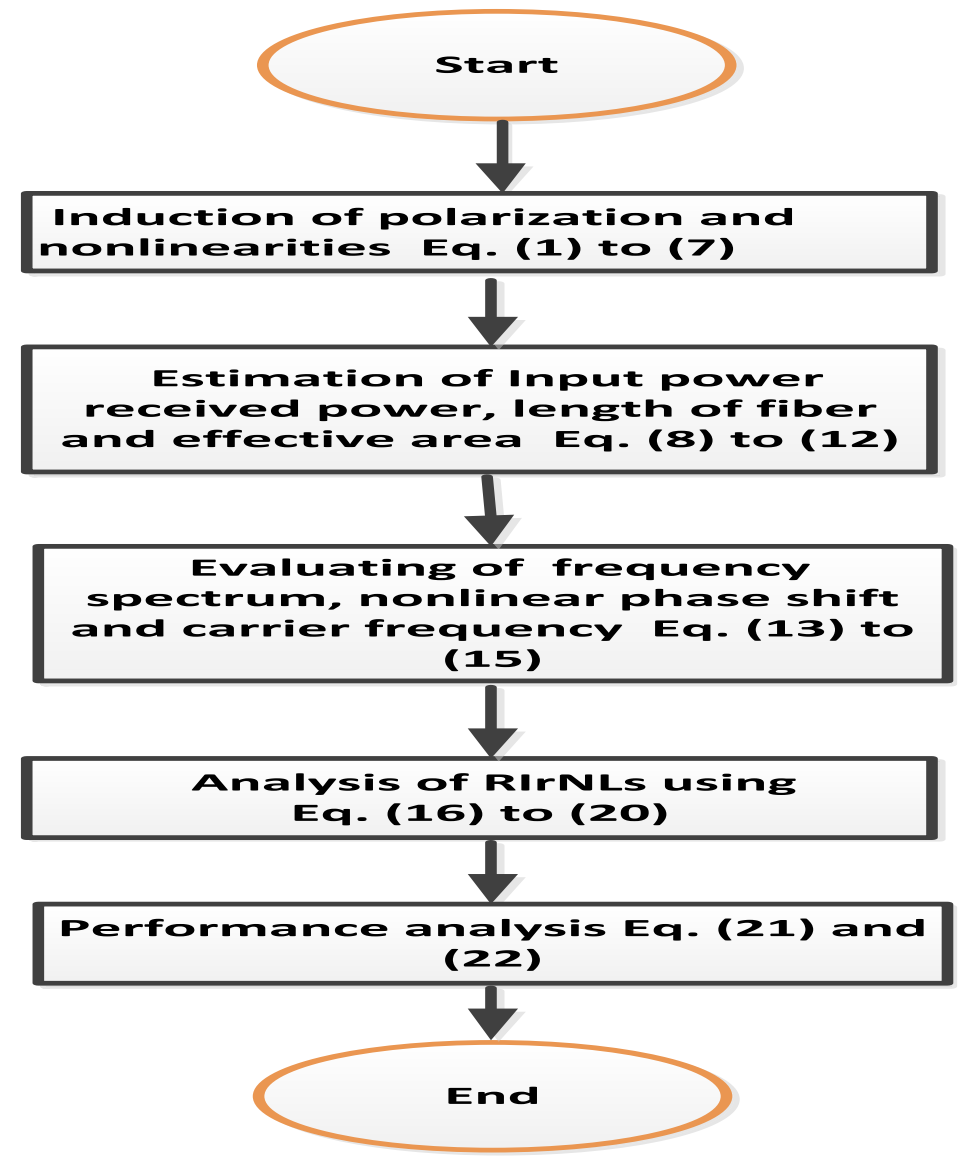

Figure 6. Proposed flow chart to how analytical and simulation models are linked.

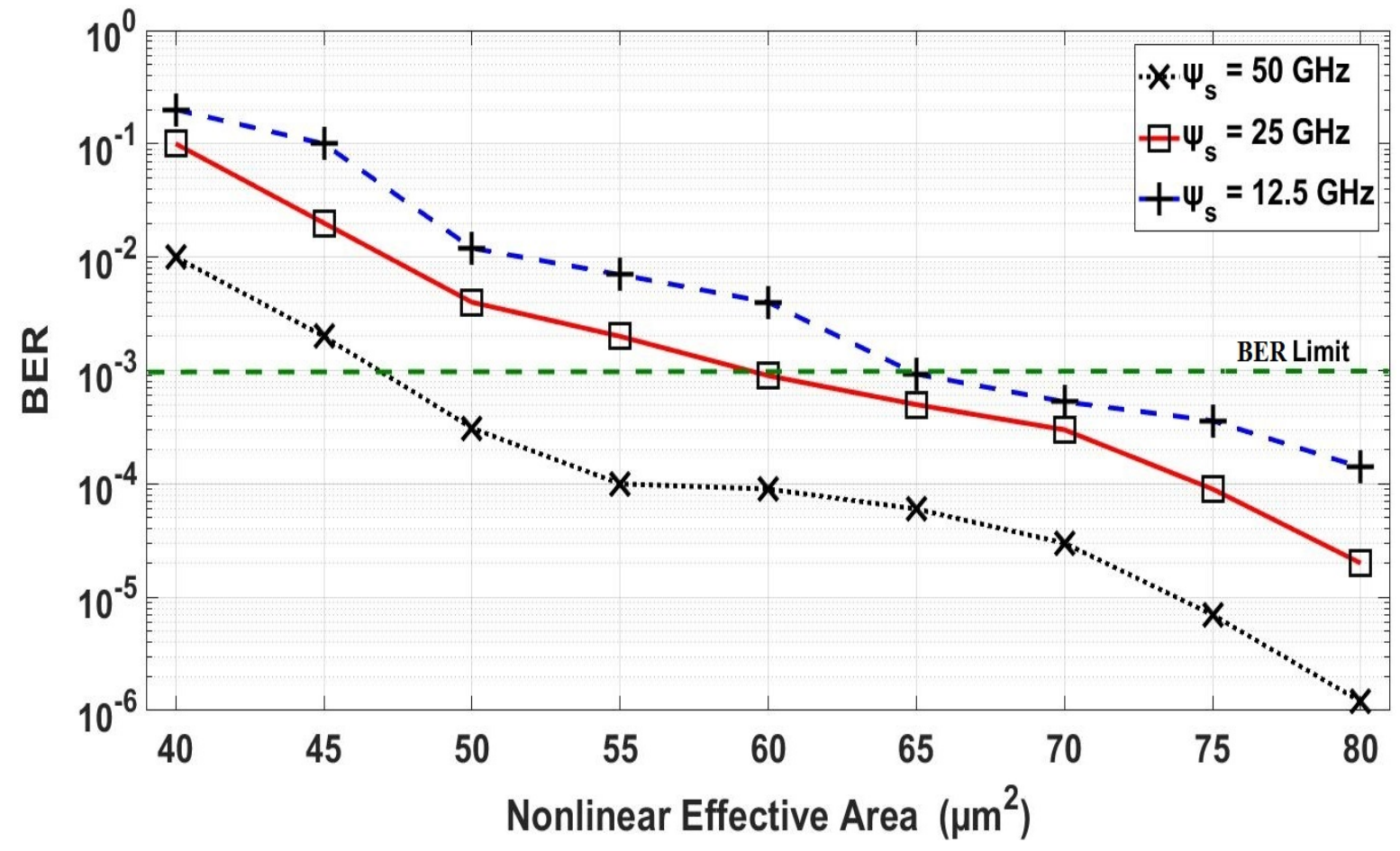

Figure 7. BER results for a range of Nonlinear effective area at 50, 25 and $12.5 \mathrm{GHz}$ channel spacings ( 32 channels). 


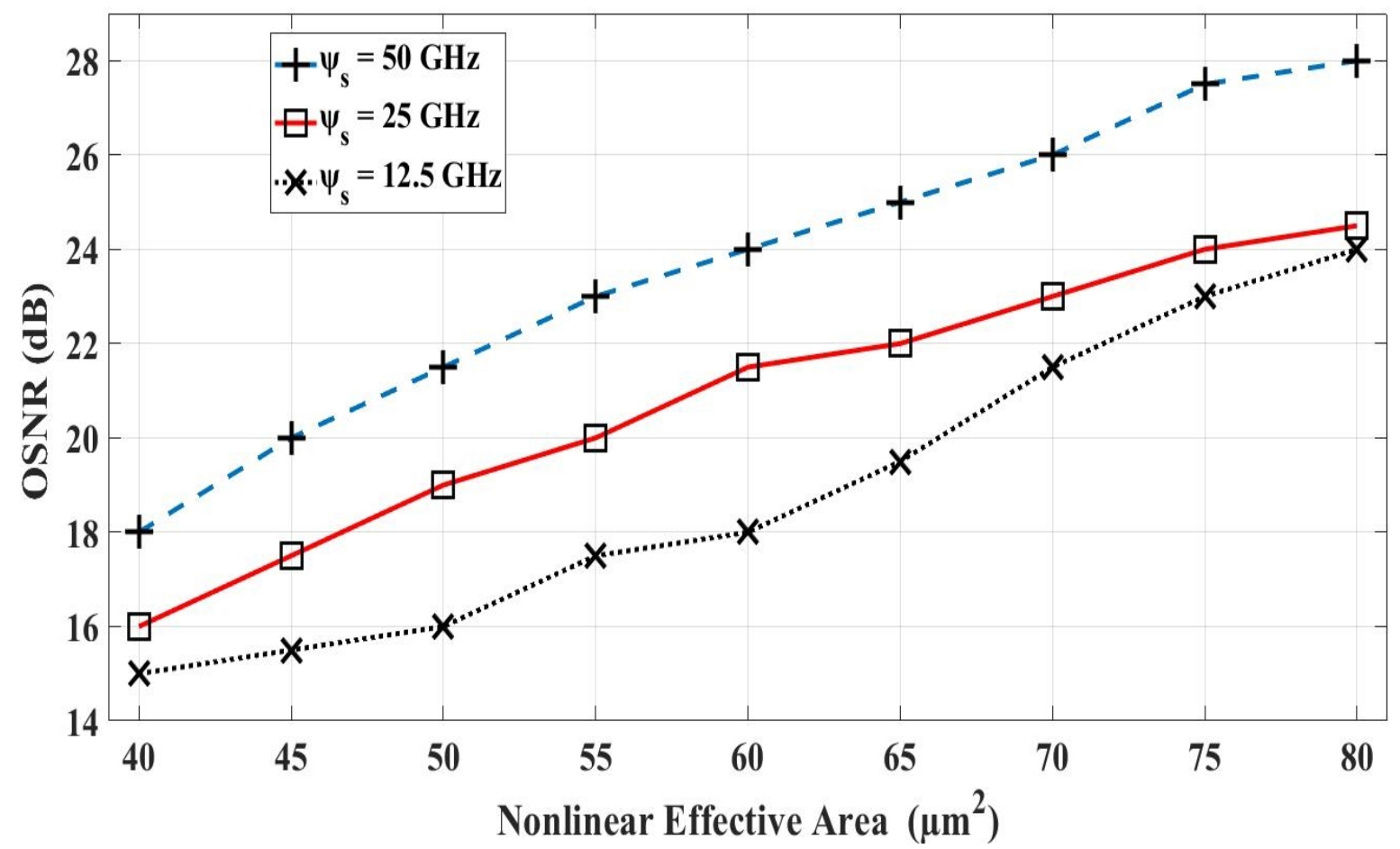

Figure 8. OSNR versus nonlinear effective area for 32 number of channels, $100 \mathrm{Gbps}$ aggregate data rate, considering different channel spacing values.

Figure 9 includes the results for BER versus effective area for different number of channels. The plot indicates that RIrNLs increase with the number of channels, for a fixed channel spacing of $50 \mathrm{GHz}$. However, the proposed system provides low BER values, even for 32 channels after the mitigation of RIrNLs effects. Moreover, the projected model examines simulation results in up to $500 \mathrm{~km}$ of fiber length as shown in Figure 10. The results also show that the proposed model has the ability to support multi channels for up to $500 \mathrm{~km}$ of optical path covered, by treating RIrNLs.

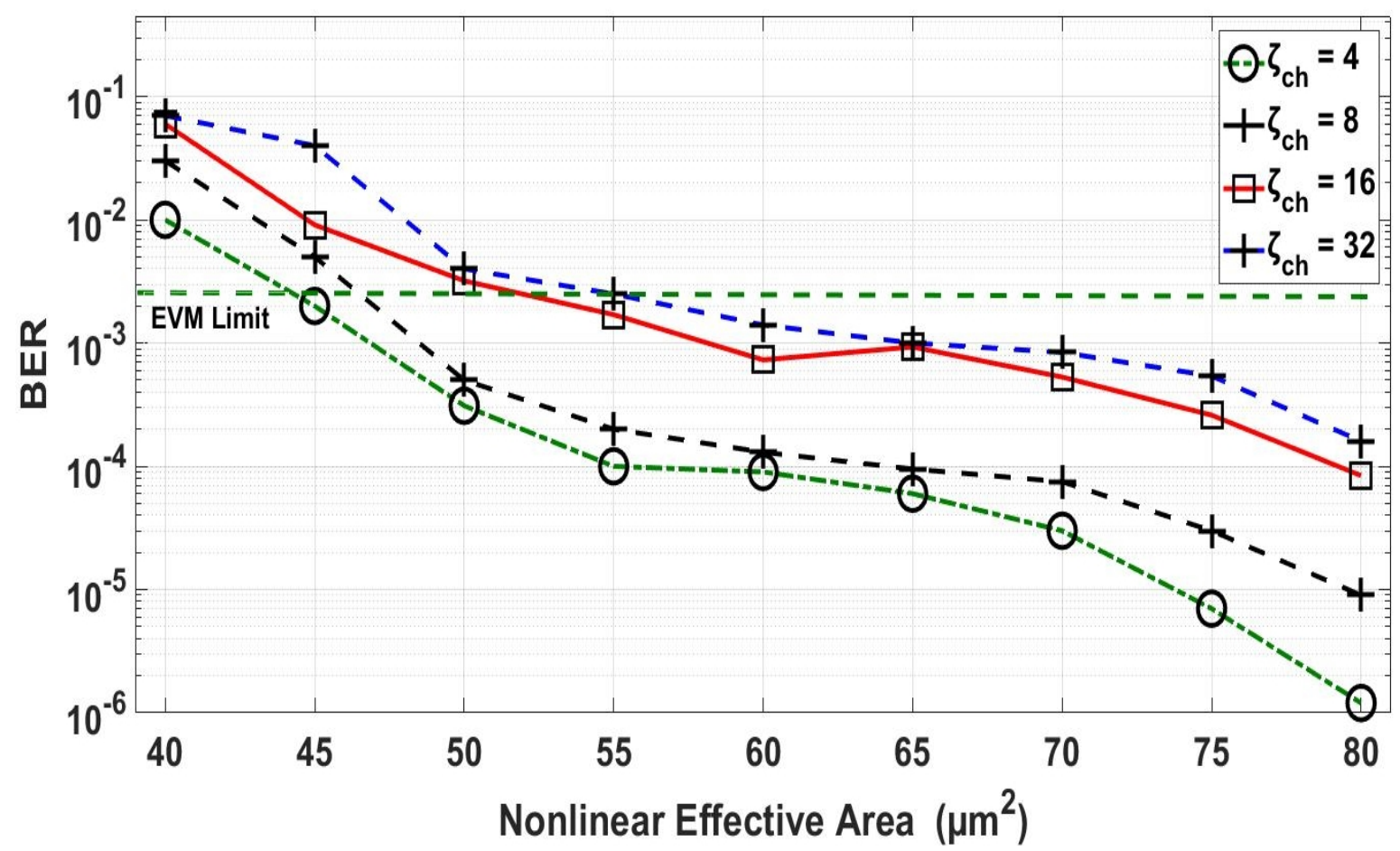

Figure 9. BER versus nonlinear effective area for 4, 8, 16 and 32 channels (50 GHz channel spacing). 


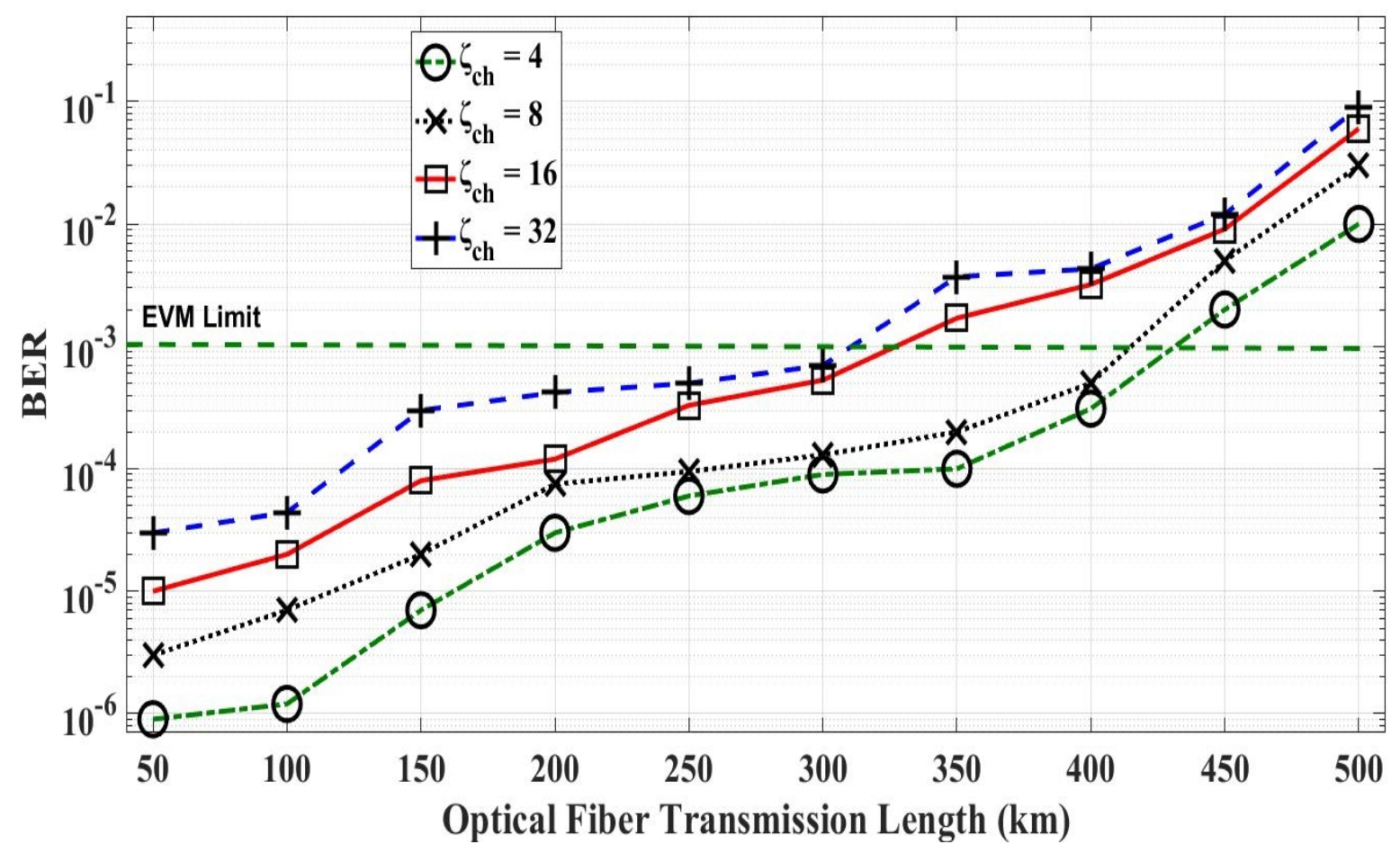

Figure 10. BER results for different fiber lengths with 4, 8, 16, and 32 channels (with $50 \mathrm{GHz}$ channel spacing).

The system performance will degrade with a reduced channel spacing. For this purpose, an additional analysis has been performed and results have been included in the paper for Dense-WDM spacings of $12.5 \mathrm{GHz}$ and $25 \mathrm{GHz}$. Since each channel carries data bandwidth of $3.16 \mathrm{GHz}$, further reduction in channel spacing causes severe distortion and very high BER. The results section has been updated with the results for $12.5 \mathrm{GHz}$ and $25 \mathrm{GHz}$ channel spacings for 32 number of total channels. The results for receiver sensitivity are shown in terms of received optical power. The BER threshold of $10^{-3}$ has been considered to analyze the receiver sensitivity. The laser frequency for first channel is $193.1 \mathrm{THz}$, which corresponds to the wavelength of $1552.5 \mathrm{~nm}$. For 32 number of channels with $25 \mathrm{GHz}$ spacings, the range for the WDM system is from $193.1 \mathrm{THz}$ to 193.875 THz. For distinct channel spacings such as $12.5,25$ and $50 \mathrm{GHz}$, the developed model is tested for different values of nonlinear refractive index and BER results are obtained, as mentioned in Figure 11. This figuredemonstrates that RIrNLs are managed efficiently at $2.6 \times 10^{-17} \mathrm{~m}^{2} / \mathrm{W}$ nonlinear refractive index. Figure 12 is obtained by performing the simulations for different data rates per channel, which shows that data rate up to $3.125 \mathrm{~Gb} / \mathrm{s}$ per user can be supported by lowest channel spacing value of $12.5 \mathrm{GHz}$, for 32 number of channels. Though an aggregate data rates of $100 \mathrm{~Gb} / \mathrm{s}(32 \times 3.125 \mathrm{~Gb} / \mathrm{s})$ is obtained for all $12.5 \mathrm{GHz}$ channel spacings, the results in Figure 12 show that higher data rates can be achieved using channel spacing of $25 \mathrm{GHz}$ or $50 \mathrm{GHz}$, as the BER performance is below the threshold for values greater than $3.125 \mathrm{~Gb} / \mathrm{s}$ user data rate.

The BER results for different input optical power levels and received power are presented in Figures 13 and 14 respectively, for different number of channels and at 100 Gbps aggregate data rate. Finally, Figure 15 shows the BER results for different values of nonlinear dispersion parameter. It is clear from the results that the lower value of nonlinear dispersion results in low range of RIrNLs. Figure 15 also presents BER for different number of channels by using $25 \mathrm{GHz}$ and $50 \mathrm{GHz}$ channel spacings at $500 \mathrm{~km}$ transmission length. Comparing the obtained results with recently published work, the 8 channel WDM system presented in [33-36] provides transmission up to $25 \mathrm{~km}$ with $25 \mathrm{GHz}$ channel spacing, in the presence of FWM non-linearities. Additionally, an advanced receiver presented in [34] enables the 8-channel WDM transmission up to $100 \mathrm{~km}$ with $100 \mathrm{GHz}$ channel spacing with BER of $5.3 \times 10^{-6}$ by mitigating the SPM, XPM and FWM non-linearities. 
Thus, the work presented in this paper is of considerable importance, as it provides lower $\mathrm{BER}$ rates for $500 \mathrm{~km}$ transmission, 32 channels and $12.5 \mathrm{GHz}$ channel spacing.

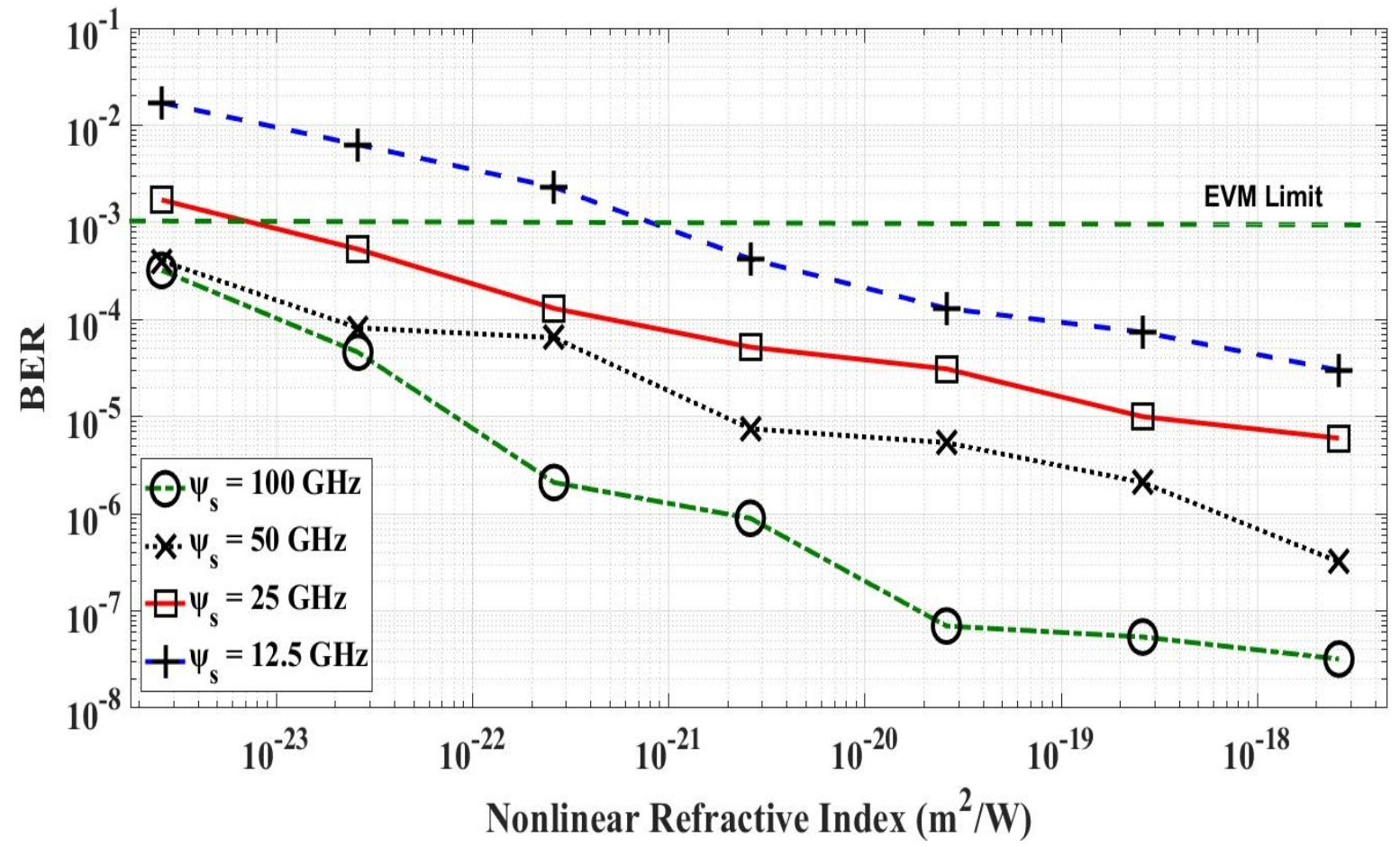

Figure 11. BER results versus refractive index for 12.5, 25, 50 and $100 \mathrm{GHz}$ channel spacing for 32 number of channels.

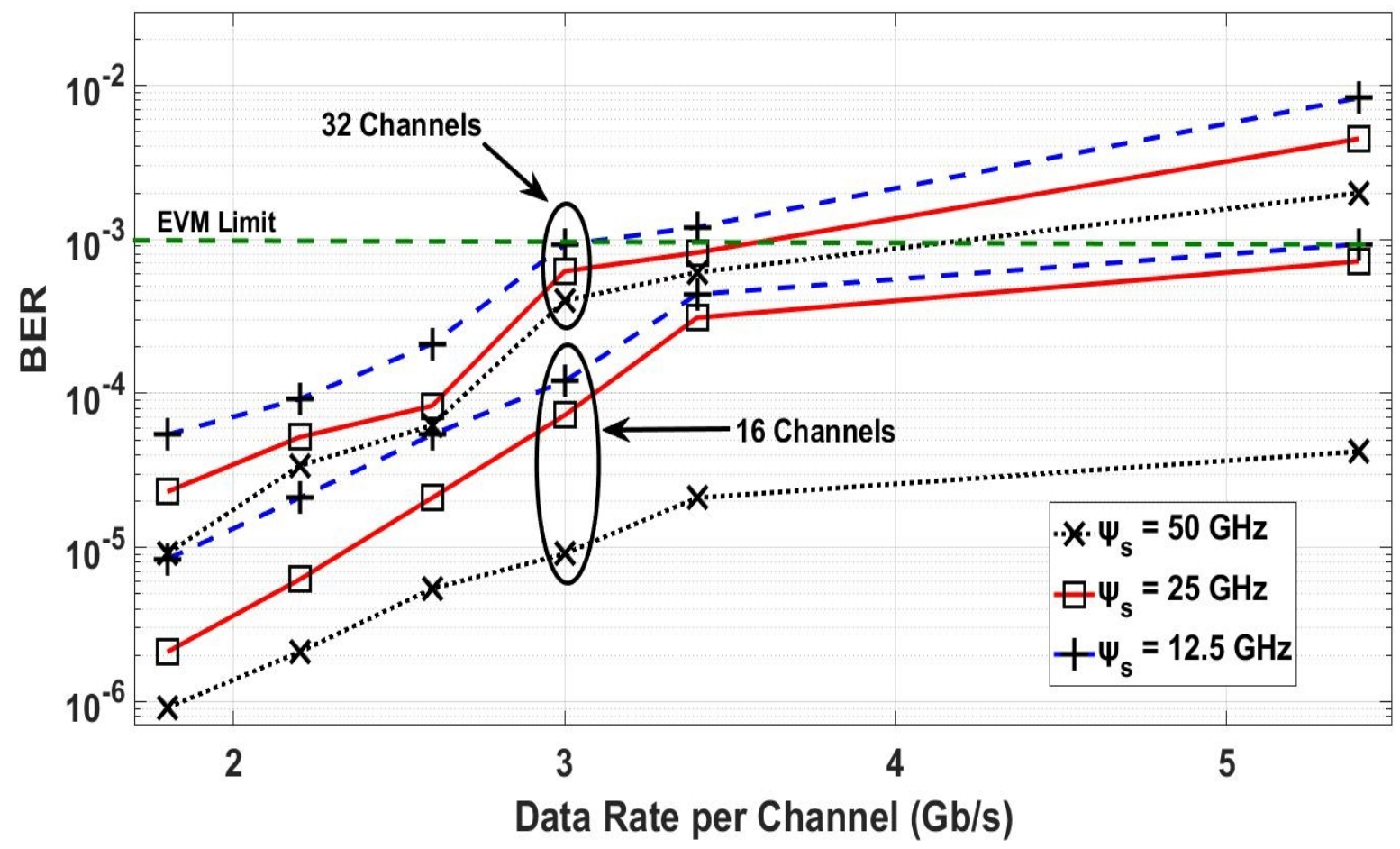

Figure 12. Data rate per channel with $12.5,25$, and $50 \mathrm{GHz}$ channel spacing, for 16 and 32 number of channels. 


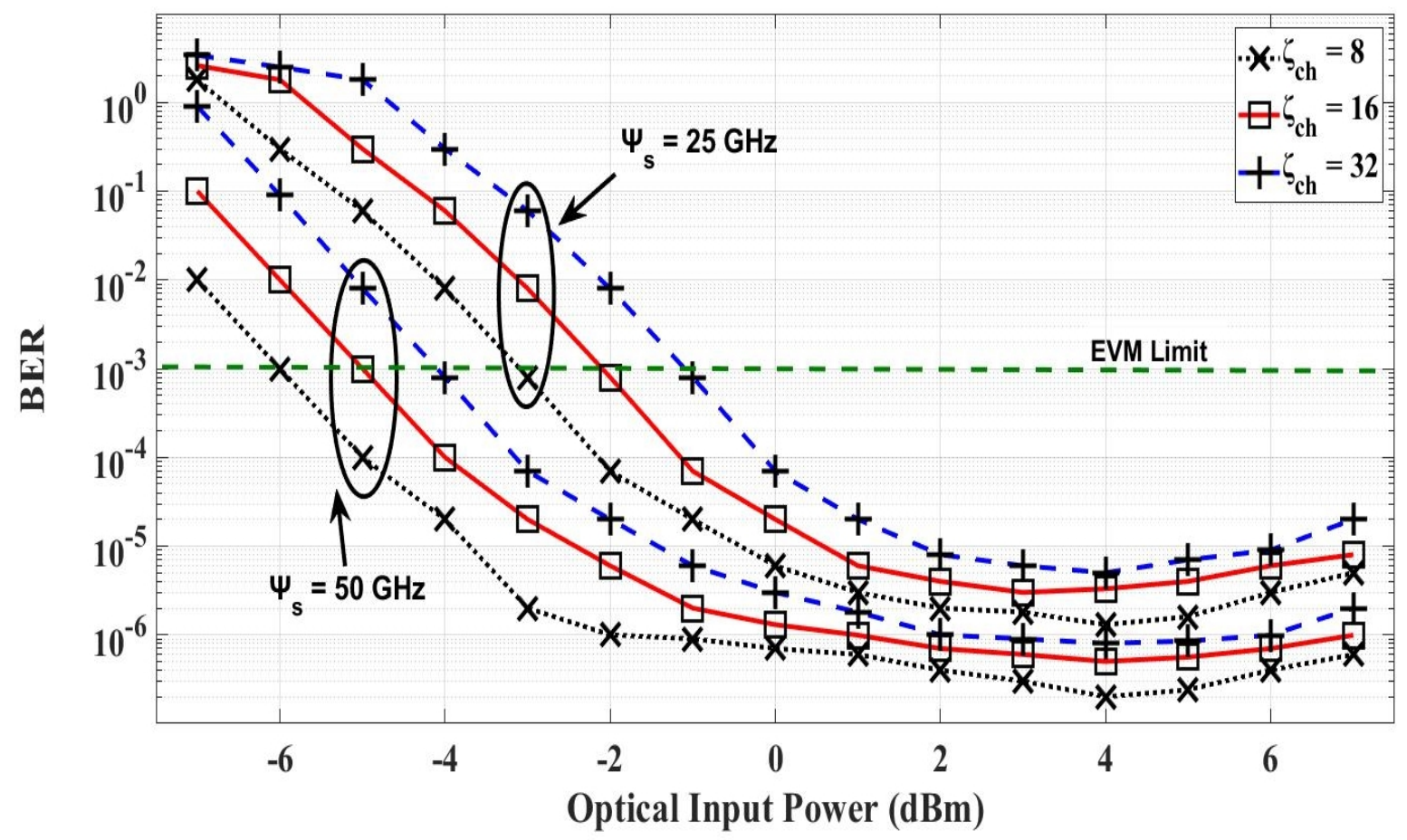

Figure 13. BER for different input power levels for 8, 16 and 32 channels with 25 and $50 \mathrm{GHz}$ channel spacing.

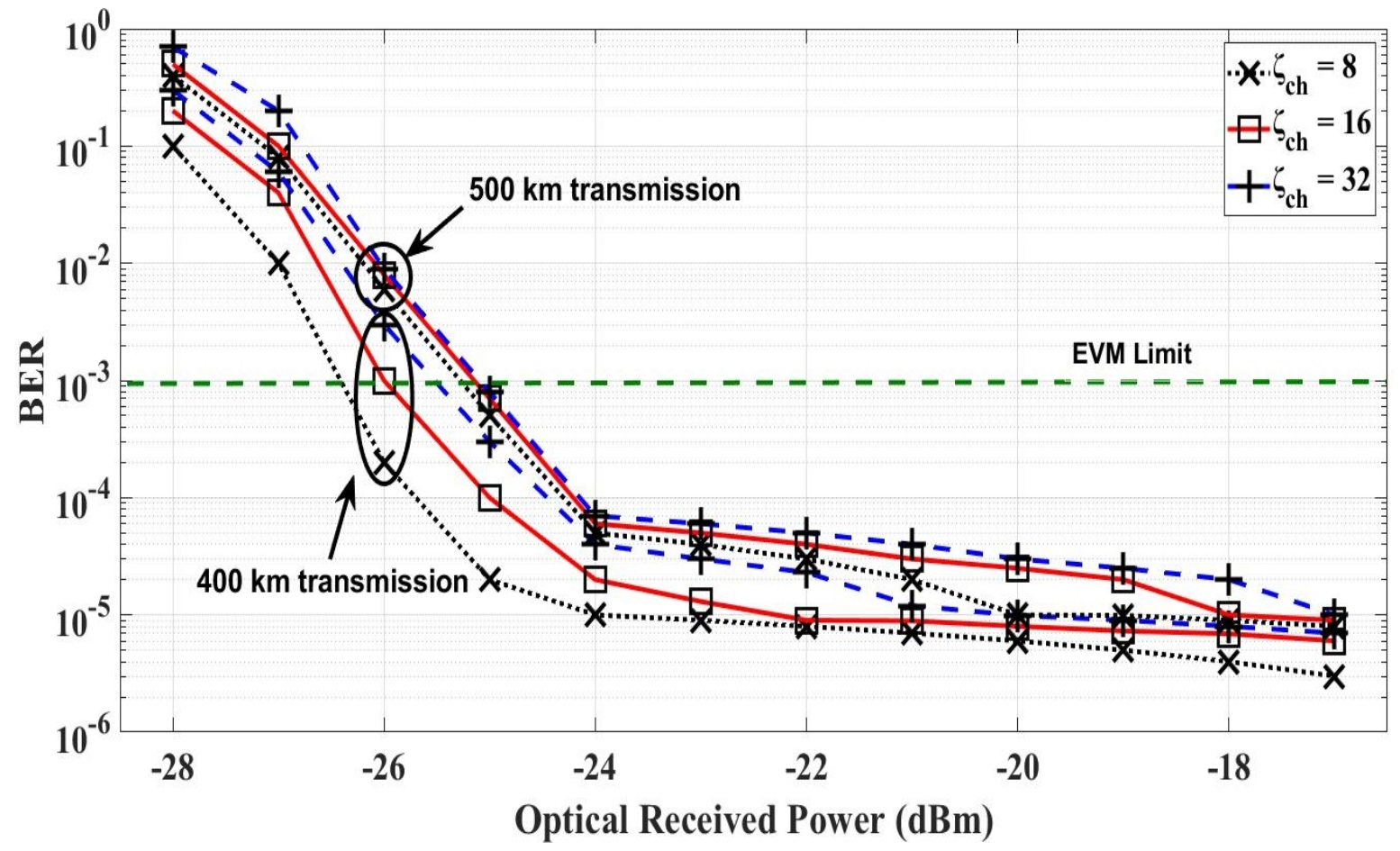

Figure 14. Receiver sensitivity for 8, 16 and 32 channels after 400 and $500 \mathrm{~km}$ of optical fiber transmission.

Comparing the obtained results with the experimental study of data transmission over $500 \mathrm{~km}$ of fiber transmission [37-40], the use of DSP for interference mitigation and compensation of non-linear effects allows one to increase the number of channels from four [37] to thirty-two channels. Similar results have been achieved as [38] without the use of polarization multiplexing, which reduces the complexity of our proposed system. The relative group delay analysis performed in [39] shows a maximum of 430 psec value, which can easily be compensated using a larger frame size during the transmission. The $13.3 \mathrm{~dB}$ 
OSNR achieved in [40] after $500 \mathrm{~km}$ transmission through a low loss fiber justifies our BER results and received eye diagram, as explained in the next paragraph.

Lastly, we explore the efficiency of the proposed model and its ability to minimize RIrNLs based on the basis of eye-diagrams for different channel spacings. Figure 16a-c shows the results for $12.5,25$, and $50 \mathrm{GHz}$ channel spacing without RIrNLs compensation at $500 \mathrm{~km}$ distance cover and $100 \mathrm{Gbps}$ aggregate data rate. While the eye diagrams after RIrNLs compensation are shown in Figure $16 \mathrm{~d}-\mathrm{f}$, which clearly demonstrates improvement in performance.

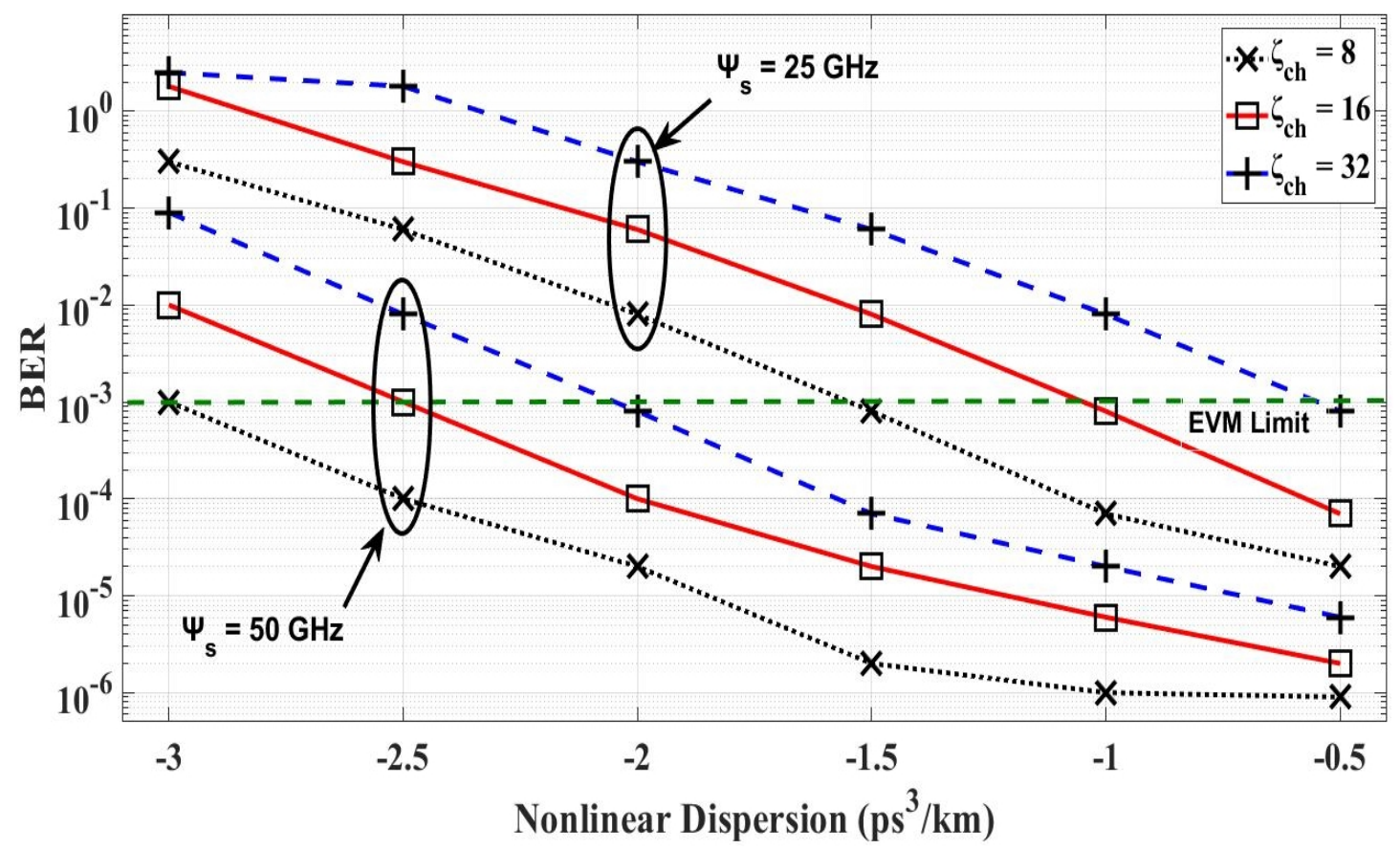

Figure 15. Nonlinear dispersion versus BER at 25 and $50 \mathrm{GHz}$ channel spacing, $500 \mathrm{~km}$ transmission distance and 8, 16 and 32 number of channels.

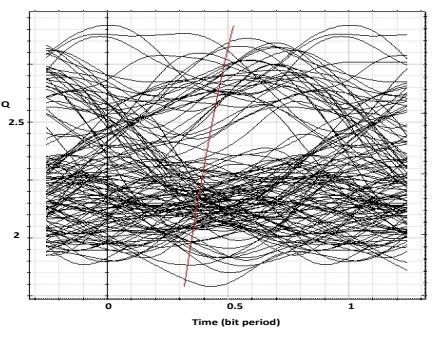

(a)

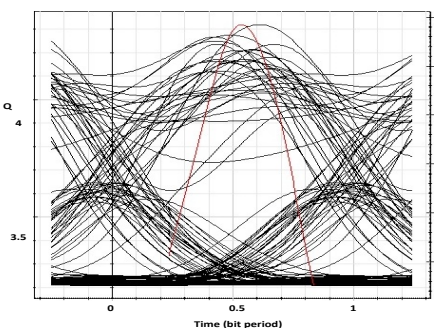

(d)

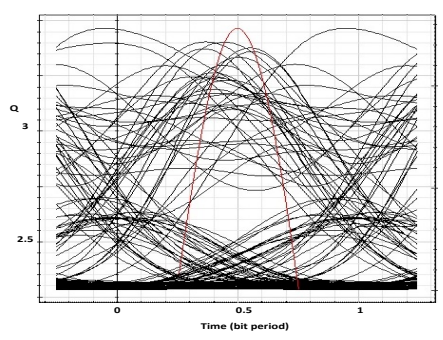

(b)

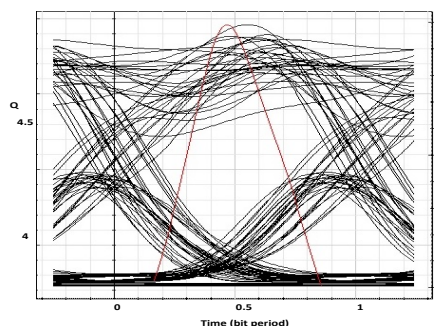

(e)

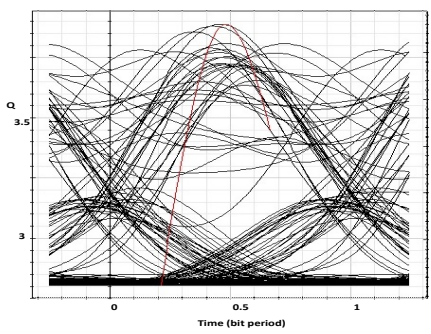

(c)

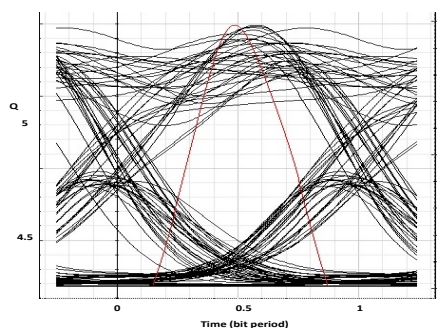

(f)

Figure 16. Results for Eye-diagrams; (a-c): For $12.5 \mathrm{GHz}, 25 \mathrm{GHz}, 50 \mathrm{GHz}$ channel spacings, respectively, without RIrNLs compensation, (d-f): For $12.5 \mathrm{GHz}, 25 \mathrm{GHz}, 50 \mathrm{GHz}$ channel spacings, respectively, after RIrNLs compensation. 


\section{Conclusions}

Mitigation of RIrNLs' effects, including XPM, FWM and SPM, are analyzed in this paper with an analytical and simulation model, in order to design an optimal OTS using FDMIMO equalizer-based enhanced DSP receiver and microstrip Chebyshev low pass filter for long-haul transmission. These impacts are investigated for distinct values of channel spacing, transmission length, number of channels, input and received power, nonlinear refractive index, nonlinear dispersion and nonlinear effective area, in a 32 channel WDM system. The simulations are performed using OSNR and BER as performance metrics. Results show that the proposed design maintains its continuity at $2.6 \times 10^{-17} \mathrm{~m}^{2} / \mathrm{W}$ refractive index, $80 \mu \mathrm{m}^{2}$ effective area, up to $500 \mathrm{~km}$ fiber length with the conventional $6 \mathrm{dBm}$ input optical power. The impacts of RIrNLs are minimized through implementation of the proposed optimum design and using duo-binary modulation, semiconductor optical amplifier and filters. The BER achieved in this work is $<10^{-5}$ till $500 \mathrm{~km}$ range, for a maximum number of 32 channels, with $100 \mathrm{Gbps}$ aggregate data rate. This shows the feasibility and effectiveness of the proposed model.

Author Contributions: Conceptualization, M.I., F.A., A.S.A. and F.M.; methodology, F.A., H.K. and M.I; software, F.A., and F.M.; validation, F.A., M.I., R.M., I.G. and F.S.A.; formal analysis, F.A., A.G., F.M., R.M. and H.K.; investigation, F.A., and F.M.; data analysis, F.A.; writing-original draft preparation, F.A.; writing-review and editing, F.M., M.I., A.S.A., R.M., F.S.A. and H.K.; visualization, F.M.; supervision, M.I. and F.A.; project administration, F.A., F.M. and M.I.; obtaining financing, M.I. and A.S.A., A.G., R.M. and F.S.A. All authors have read and agreed to the published version of the manuscript.

Funding: EMMAT E-mobility and sustainable materials and technologies (E-mobilność oraz zrównoważone materiały i technologie) Projekt nr PPI/APM/2018/1/00027.

Institutional Review Board Statement: Not applicable.

Informed Consent Statement: Not applicable.

Data Availability Statement: No new data were created or analyzed in this study. Data sharing is not applicable to this article.

Acknowledgments: This work was supported by the Ministry of Education Kingdom of Saudi Arabia and the Deanship of Scientific Research, Najran University Kingdom of Saudi Arabia, under code number NU/ESCI/17/007.

Conflicts of Interest: The authors declare no conflict of interest.

\section{References}

1. Ali, F.; Khan, Y.; Muhammad, F.; Habib, U.; Abbas, Z.H.; Khan, M.A.; Ali, A. Extenuation of phase shift influenced nonlinear impairments in fiber optics network. Trans. Emerg. Telecommun. Technol. 2020, 31, e3930. [CrossRef]

2. Muhammad, F.; Ali, F.; Habib, U.; Usman, M.; Khan, I.; Kim, S. Time domain equalization and digital back-propagation method-based receiver for fiber optic communication systems. Int. J. Opt. 2020, 2020, 3146374. [CrossRef]

3. Ali, F.; Ahmad, S.; Muhammad, F.; Habib, U.; Kim, S. Adaptive equalization for dispersion mitigation in multi-channel optical communication network. Electronic 2019, 8, 1364. [CrossRef]

4. Xu, T.; Shevchenko, N.A.; Semrau, D.; Liga, G.; Alvarado, A.; Killey, R.I.; Bayvel, P. Modulation format dependence of digital nonlinearity compensation performance in optical fibre communication systems. Opt. Express 2017, 25, 3311-3326. [CrossRef] [PubMed]

5. Agrell, E.; Karlsson, M.; Chraplyvy, A.R.; Richardson, D.J.; Krummrich, P.M.; Winzer, P.; Roberts, K.; Fischer, J.K.; Savory, S.J.; Eggleton, B.J.; et al. Roadmap of optical communications. J. Opt. 2016, 18, 063002. [CrossRef]

6. Cartledge, J.C.; Guiomar, F.P.; Kschischang, F.R.; Liga, G.; Yankov, M.P. Digital signal processing for fiber nonlinearities. Opt. Express 2017, 25, 1916-1936. [CrossRef] [PubMed]

7. Bakhshali, A.; Chan, W.Y.; Cartledge, J.C.; Sullivan, M.O.; Laperle, C.; Borowiec, A.; Roberts, K. Frequency-domain Volterra-based equalization structures for efficient mitigation of intrachannel Kerr nonlinearities. J. Lightwave Technol. 2016, 34, 1770-1777. [CrossRef]

8. Liu, X.; Chraplyvy, A.; Winzer, P.; Tkach, R.; Chandrasekhar, S. Phase-conjugated twin waves for communication beyond the Kerr nonlinearity limit. Nat. Photonics 2013, 7, 560. [CrossRef] 
9. Habib, U.; Aighobahi, A.E.; Nair, M.; Zhu, H.; Quinlan, T.; Walker, S.D.; Gomes, N.J. Performance improvement for OFDM-RoF transported $60 \mathrm{GHz}$ system using spatial diversity and multiplexing. In Proceedings of the IEEE International Conference on Communications Workshops (ICC Workshops), Paris, France, 21-25 May 2017; pp. 211-216.

10. Habib, U.; Steeg, M.; Stöhr, M.A.; Gomes, N.J. Radio-over-fiber-supported 60GHz multiuser transmission using leaky wave antenna. In Proceedings of the IEEE International Topical Meeting on Microwave Photonics (MWP), Beijing, China, 23-26 October 2017; pp. 1-4.

11. Ali, F.; Muhammad, F.; Habib, U.; Khan, Y.; Usman, M. Modeling and minimization of FWM effects in DWDM-based long-haul optical communication systems. Photon Netw. Commun. 2020. [CrossRef]

12. Kishikawa, H.; Uetai, M.; Gotoi, N. All-Optical Modulation Format Conversion between OOK, QPSK, and 8QAM. J. Lightwave Technol. 2019, 37, 3925-3931. [CrossRef]

13. Liga, G.; Saavedra, G.; Bayvel, P. Combining Optical Phase Conjugation and Volterra Equalisation: A Novel Nonlinearity Compensation Scheme. J. Lightwave Technol. 2018, 36, 377-400.

14. Rademacher, G.; Luis, R.S.; Puttnam, B.J.; Maruyama, R.; Aikawa, K.; Awaji, Y.; Furukawa, H.; Petermann, K.; Wada, N. Investigation of Intermodal Nonlinear Signal Distortions in Few-Mode Fiber Transmission. J. Lightwave Technol. 2019, 37, 1273-1279. [CrossRef]

15. Kong, M.; Kong, M.; Li, X.; Zhang, J.; Xin, K.W.X.; Zhao, F.; Yu, J. High spectral efficiency 400 Gb/s transmission by different modulation formats and advanced DSP. J. Lightwave Technol. 2019, 37, 5317-5325. [CrossRef]

16. Al-Rawachy, E.; Giddings, R.P.; Tang, J. Experimental demonstration of a real-time digital filtermultiple access PON with low complexity DSP-based interference cancellation. J. Lightwave Technol. 2019, 37, 4315-4329. [CrossRef]

17. Giacoumidis, E.; Lin, Y.; Wei, J.; Aldaya, I.; Tsokanos, A.; Barry, L.P. Harnessing machine learning for fiber-induced nonlinearity mitigation in long-haul coherent optical OFDM. Future Internet 2018, 11, 2. [CrossRef]

18. Chen, Y.; Shen, S.; Zhou, Q.; Yao, S.; Zhang, R.; Omar, S. A reliable OFDM-based MMW mobile fronthaul with DSP-aided sub-band spreading and time-confined windowing. J. Lightwave Technol. 2019, 37, 3236-3243. [CrossRef]

19. Guiomar, F.P.; Carena, A.; Bosco, G.; Bertignono, L.; Nespola, A.; Poggiolini, P. Nonlinear mitigation on subcarrier-multiplexed PM-16QAM optical systems. Opt. Express 2017, 25, 4298-4311. [CrossRef]

20. Ali, F.; Khan, Y.; Ali, A.; Ahmad, G. Minimization of nonlinear impairments and its impact on transmission performances of high-capacity long-haul optical networks. J. Opt. Commun. 2018. [CrossRef]

21. Qamar, F.; Islam, M.K.; Ali, S.Z.; Ali, M. Secure duobinary signal transmission in optical communication networks for high performance \& reliability. IEEE Access 2018, 5, 17795-17802.

22. Zhang, H.; Batshon, H.G.; Davidson, C.R.; Foursa, D.G.; Pilipetskii, A. Multi dimensional coded modulation in long-haul fiber optic transmission. J. Lightwave Technol. 2015, 33, 2876-2883. [CrossRef]

23. Stojanovic, N.; Changsong, X. An efficient method for skew estimation and compensation in coherent receivers. IEEE Photonics Technol. Lett. 2016, 28, 489-492. [CrossRef]

24. Benyahya, K.; Simonneau, C.; Ghazisaeidi, A.; Barr, N.; Jian, P.; Morizur, J.F.; Labroille, G.; Bigot, M.; Sillard, P.; Provost, J.G.; et al. Multiterabit Transmission Over OM2 Multimode Fiber with Wavelength and Mode Group Multiplexing and Direct Detection. J. Light. Technol. 2018, 36, 355-360. [CrossRef]

25. Zhuge, Q.; Chen, X. Advances in modulation and DSP for optical transmission systems. J. Opt. Commun. 2018, 409, 1-136. [CrossRef]

26. Maharana, D.; Rout, R. A 4 channel WDM based hybrid optical Fiber/FSO communication system using DP QPSK modulation for bit rate of 100/112 Gb/s. Int. J. Eng. Res. Technol. 2019, 8, 442-445.

27. Obaid, H.M.; Shahid, H. Achieving high gain using Er-Yb codoped waveguide/fiber optical parametric hybrid amplifier for dense wavelength division multiplexed system. Opt. Eng. 2018, 57, 056108. [CrossRef]

28. Niaz, A.; Qamar, F.; Islam, K.; Shahzad, A.; Shahzadi, R.; Ali, M. Performance analysis and comparison of QPSK and DP-QPSK based on optical fiber communication systems. ITEE J. 2018, 7, 34-39.

29. Tipsuwannakul, E.; Li, J.; Karlsson, M.; Andrekson, P.A. Performance comparisons of DP16-QAM and duobinary-shaped DP-QPSK for optical systems with 4.1 Bit/s/Hz spectral efficiency. J. Lightwave Technol. 2012, 30, 2307-2314. [CrossRef]

30. El-Naha, F.I. Coherent quadrature phase shift keying optical communication systems. Optoelectron. Lett. 2018, 14, 372-375. [CrossRef]

31. Kahn, J.M.; Miller, D.A.B. Communications expands its space. Nat. Photonics 2017, 11, 5-8. [CrossRef]

32. Perin, J.K.; Shastri, A.; Kahn, J. Design of low-power DSP-free coherent receivers for data center links. J. Lightwave Technol. 2017, 35, 4650-4662. [CrossRef]

33. Miao, X.; Bi, M.; Fu, Y.; Li, L.; Hu, W. Experimental study of NRZ, Duobinary, and PAM-4 in O-band DML-based on 100G-EPON. IEEE Photonics Technol. Lett. 2017, 29, 1490-1493. [CrossRef]

34. Dong, Y.; Al-Rawachy, E.; Giddings, R.P.; Jin, W.; Nesset, D.; Tang, J.M. Multiple channel interference cancellation of digital filter multiple access PONs. J. Lightwave Technol. 2017, 35, 34-44. [CrossRef]

35. Manzoor, H.U.; Zafar, M.; Manzoor, S.U.; Khan, T.; Liu, S.; Manzoor, T.; Saleem, S.; Kim, W.Y.; Alih, M. Improving FWM efficiency in Bi-directional ultra DWDM-PON networking centered light source by using PMD emulator. Results Phys. 2020, 16, 102922. [CrossRef] 
36. De Sousa, F.B.; Oliveira, J.E.D.; Sabino, E.R.; Sousa, F.M.D.; Costa, M.B.C. Simulations of SPM, XPM and FWM in Single-Mode Fiber Optic Networks. In Proceedings of the SEMINATEC XII Workshop on Semiconductors and Micro and Nano Technology, Sao Paulo, Brazil, 27-28 April 2017.

37. Kim, H.K.; Chandrasekhar, S.; Srivastava, A.; Burrus, C.A.; Buhl, L. 10 Gbit/s based WDM signal transmission over 500 km of NZDSF using semiconductor optical amplifier as the in-line amplifier. Electron. Lett. 2001, 37, 185-187. [CrossRef]

38. Gainov, V.; Gurkin, N.; Lukinih, S.; Makovejs, S.; Akopov, S.; Ten, S.; Nanii, O.; Treshchikov, V.; Sleptsov, M. Record 500 km unrepeatered $1 \mathrm{Tbit} / \mathrm{s}(10 \times 100 \mathrm{G})$ transmission over an ultra-low loss fiber. Opt. Express 2014, 22, 22308-22313. [CrossRef] [PubMed]

39. Sahara, A.; Komukai, T.; Yamada, E.; Nakazawa, M. 40 Gbit/s return-to-zero transmission over 500 km of standard fiber using chirped fiber Bragg gratings with small group delay ripples. In Optical Fiber Communication Conference; Optical Society of America: San Diego, CA, USA, 2001.

40. Gainov, V.V.; Gurkin, N.V.; Lukinih, S.N.; Shikhaliev, I.I.; Skvortsov, P.I.; Makovejs, S.; Akopov, S.G.; Ten, S.Y.; Nanii, O.E.; Treshchikov, V.N. 500 km unrepeatered 200 Gbits-1 transmission over a G. 652-compliant ultra-low loss fiber only. Laser Phys. Lett. 2015, 12, 066201. [CrossRef] 